\title{
A Case of Massive Hepatic Infarction in a Patient with HELLP Syndrome
}

\author{
Jessica Morgan, MD ${ }^{1} \quad$ Micaela Della Torre, MD, MS² Anna R. Whelan, MD ${ }^{1} \quad$ Sophia M. Rodriguez, MD ${ }^{1}$ \\ Laura M. DiGiovanni, MD² \\ ${ }^{1}$ Department of Obstetrics and Gynecology, The University of Illinois \\ at Chicago, Chicago, Illinois \\ 2 Division of Maternal-Fetal Medicine, Department Obstetrics and \\ Gynecology, The University of Illinois at Chicago, Chicago, Illinois \\ Address for correspondence Jessica Morgan, MD, Department of \\ Obstetrics and Gynecology, The University of Illinois at Chicago, 820 \\ South Wood Street, MC 808, Chicago, IL 60612 \\ (e-mail: jmorga36@uic.edu).
}

Am J Perinatol Rep 2019;9:e84-e87.

\begin{abstract}
\section{Keywords}

- HELLP syndrome

- pre-eclampsia

- liver

- infarction

- preterm

Background Hepatic infarction is an exceedingly rare complication of hemolysis, elevated liver enzymes, and low platelets syndrome. Few cases have been described in the medical literature and the true incidence remains unknown. It can lead to fulminant liver failure, liver transplant, or death if not promptly addressed.

Case Report A 22-year-old primigravida presented with right upper quadrant and epigastric pain at 28 weeks' gestation. She had severely elevated blood pressures requiring intravenous antihypertensives as well as proteinuria, thrombocytopenia, and mild transaminitis. Within 6 hours of admission, her rapidly rising liver function tests (LFTs) necessitated urgent delivery by primary cesarean section. Her liver enzymes continued to rapidly worsen postoperatively and immediate postpartum computed tomography of the abdomen and pelvis revealed massive hepatic infarction, $11 \times 10 \times 15 \mathrm{~cm}$, of the right lobe of the liver. Her transaminases peaked at alanine transferase of $2,863 \mathrm{IU} / \mathrm{L}$ and aspartate transferase of 2,732 IU/L. She received supportive multidisciplinary intensive care, and LFTs returned to normal by postoperative day 20 .

Conclusion Hepatic infarction is an extraordinarily rare complication of pre-eclampsia. Early recognition and prompt multidisciplinary management are vital to prevent catastrophic bleeding, hepatic failure, and death.
\end{abstract}

Hepatic infarction is a known, yet exceedingly rare, complication of hemolysis, elevated liver enzymes, and low platelets (HELLP) syndrome. It is often described in patients with antiphospholipid syndrome (APLS). APLS is an autoimmune disorder that can cause serious adverse obstetric outcomes in women. Complications include recurrent spontaneous abortion, preterm delivery, pre-eclampsia, intrauterine growth restriction, HELLP syndrome, and other abnormalities of placentation. Outside of pregnancy women with APLS may experience spontaneous thromboembolic events including deep vein thrombosis and pulmonary

(1DDr. Morgan's ORCID is http://orcid.org/0000-0002-3253-8804. embolism. Diagnosis of APLS is typically performed at the time of the inciting event-blood clot, pulmonary embolism, miscarriage, preterm delivery-and then again 12 weeks later for confirmation. Specific autoantibodies tested include anticardiolipin immunoglobulin $\mathrm{G}(\operatorname{IgG})$ and immunoglobulin $\mathrm{M}(\operatorname{IgM})$, lupus anticoagulant, and anti- $\beta 2$ glycoprotein $\operatorname{IgG}$ and $\operatorname{IgM}$.

Women with previously diagnosed APLS are often started on anticoagulation when they become pregnant, with a lowmolecular-weight heparin and/or aspirin. However, many women are unaware that they have a diagnosis of APLS until they experience an initial thrombotic event or an adverse obstetric outcome, often early miscarriage. Even with received

January 8, 2019 accepted after revision January 21, 2019
Dol https://doi.org/

10.1055/s-0039-1681028. ISSN 2157-6998.
Copyright (c) 2019 by Thieme Medical

Publishers, Inc., 333 Seventh Avenue, New York, NY 10001, USA. Tel: +1(212) 584-4662
License terms

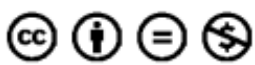


treatment, women with APLS can still develop serious obstetrical complications including pre-eclampsia, eclampsia, and HELLP syndrome. These can be life threatening to both mother and infant.

Even without the diagnosis of APLS, it is possible to develop HELLP syndrome with no prior pathology. The catastrophic complications of HELLP syndrome still exist without the underlying pathophysiology of APLS. Women with HELLP syndrome can quickly develop disseminated intravascular coagulopathy (DIC) requiring transfusion of blood, platelets, clotting factors, and even plasma exchange. ${ }^{1}$ Although HELLP syndrome is a rare event in the general population, it is important to understand because of the high risk of loss of maternal life. APLS has long been believed to be only a thrombotic disease but may also have a microangiopathic component which can explain its correlation with HELLP syndrome. HELLP syndrome is noted to occur almost exclusively in the third trimester, and very rarely before 27 weeks' gestation. $^{2}$

Our case represents a patient at the earlier end of expected gestational ages to experience HELLP syndrome, with no prior pregnancy or thrombotic event to suggest a maternal risk of HELLP syndrome such as APLS. Patients perceived to be at low risk may suffer the most from obstetrical emergencies as we are not expecting them to happen. In the absence of prior testing, it is possible to experience a major obstetrical emergency such as HELLP syndrome without any prior suggestion of pathology, as was the case in our patient.

\section{Case Report}

The patient is a 22-year-old, gravida one, para zero at 28 weeks and 5 days of gestation presented to an outside hospital for severe abdominal pain, nausea, and vomiting in the early morning hours. On presentation she was found to have severe range blood pressures with a maximum pressure of $218 / 110 \mathrm{~mm} \mathrm{Hg}$. She required multiple intravenous (IV) doses of hydralazine and labetalol to bring down her blood pressure, and was started on magnesium sulfate for seizure prophylaxis. She also was noted to have abnormal laboratories including a spot protein-creatinine ratio of 6,703 and aspart aminotransferase (AST) of 202 IU/L and alanine aminotransferase (ALT) of $180 \mathrm{IU} / \mathrm{L}$. At this time, her platelets were $195,000 / \mu \mathrm{L}$. Due to concern for pre-eclampsia with severe features and imminent preterm delivery, she received betamethasone for fetal lung maturity and the decision was made to transfer to a tertiary-care center.

By the time she arrived at our center, her blood pressures were again severely elevated and her liver enzymes had tripled. She was quickly treated with multiple doses of IV labetalol and PO nifedipine, without effect. Along with this newly refractory severe hypertension, the patient was also found to have a sharp increase in AST to 1,195 IU/L and ALT to $909 \mathrm{IU} / \mathrm{L}$, as well as drop in platelets to $105,000 / \mu \mathrm{L}$. Decision was made for immediate cesarean delivery for HELLP syndrome.

Cesarean delivery was uncomplicated with estimated blood loss of $600 \mathrm{~mL}$. Her blood pressure stabilized after delivery. However, her transaminases continued to rise. Within 6 hours of delivery, her AST rose to 1,620 IU/L and ALT to 1,636 IU/L. Overnight, they continued to rise, with AST reaching 2,065 $\mathrm{IU} / \mathrm{L}$ and ALT at 2,380 $\mathrm{IU} / \mathrm{L}$. Her international normalized ratio also rose to 1.4 from an initial level of 1.1 on admission. Her platelets also dropped to $36,000 / \mu \mathrm{L}$ overnight. She continued to have right upper quadrant pain and right subscapular pain. Computed tomography (CT) scan of the abdomen and pelvis was performed and revealed a 10 $\mathrm{cm} \times 10 \mathrm{~cm} \times 14 \mathrm{~cm}$ area of hyperattenuation on the right hepatic lobe concerning for ischemia versus infarction (-Fig. 1). Transplant surgery was consulted, as well as the intensive care unit. Per transplant recommendations, the patient underwent right upper quadrant ultrasound with Doppler, which was again consistent with right hepatic infarction or ischemia. The patient was transferred to the Medical Intensive Care Unit (MICU). Liver function tests (LFTs) continued to rise and her blood counts continued to drop. She was ultimately transfused two units of packed red blood cells and two units of fresh-frozen plasma. Her model for end-stage liver disease score was calculated at 20 and the patient underwent a series of tests including antiphospholipid panel, inherited thrombophilias, and hepatitis panel all of which were within accepted parameters. LFTs began to downtrend by postoperative day (POD) 3 and continued to do so during her admission. On discharge, her AST had normalized and ALT was still elevated at 397 IU/L on POD 8. The patient had close outpatient follow-up with maternal-fetal medicine and hepatology. Laboratories were trended and were found to have normalized at an outpatient clinic visit on POD 20. She was seen by hepatology outpatient twice within a month from delivery with normal LFTs, and planned to repeat LFTs in 3 months. Their notes state that they expect some areas of infarction to regenerate and others to be replaced by fibrous tissue, with some potential for longterm consequences of ischemic cholangiopathy and chronic liver disease. They continue to recommend regular followup. Patient planned to have repeat APLS laboratories at 12 weeks after first set but was lost to follow-up.

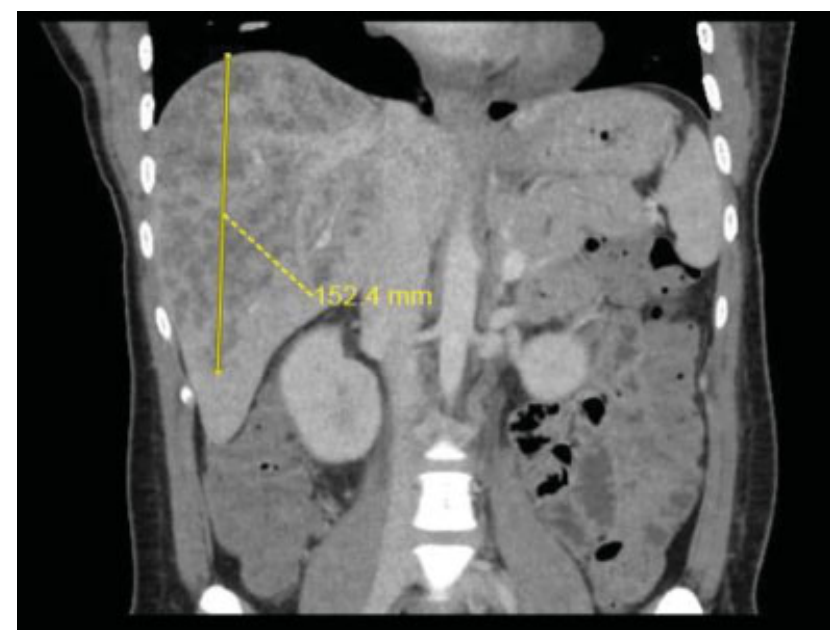

Fig. 1 Computed tomography of the abdomen and pelvis showing $60 \%$ infarction of right hepatic lobe. 


\section{Discussion}

HELLP syndrome is a potential complication of pregnancies, often associated with severe pre-eclampsia. HELLP syndrome has been estimated to complicate 0.5 to $0.9 \%$ of all pregnancies, and 10 to $20 \%$ of all cases of pre-eclampsia with severe features. ${ }^{3}$ The diagnosis of pre-eclampsia with severe features involves extreme elevations in blood pressure combined with evidence of end-organ damage. Approximately $10 \%$ of pregnant women with severe pre-eclampsia will develop abnormal LFTs. HELLP syndrome occurs within 48 hours of delivery, $20 \%$ of the time. ${ }^{4,12}$ A majority of these cases resolve spontaneously, without lasting damage to the liver. A concerning symptom that some women may experience in the setting of pre-eclampsia with impending liver damage is epigastric pain, or more specifically, right upper quadrant pain. ${ }^{3}$ A major cause of concern when this symptom is detected is that a hematoma may be developing within the liver capsule. Potentially, the most catastrophic outcome of this would be hepatic rupture, which can lead to maternal death. ${ }^{1}$ Several case reports have described women with HELLP syndrome who ultimately suffered hepatic infarction, evidenced on imaging studies. When hepatic damage is extensive enough, some of these women have undergone hepatic transplantation. From October 1987 to December 2006, there have been eight liver transplants in the United States performed for the indication of HELLP syndrome, often with the complication of liver necrosis and liver failure following hepatic rupture. Worldwide, there are also case reports of hepatic transplant as the ultimate treatment for HELLP syndrome's most devastating complications.

The mainstay of supportive management includes transfusion of blood products as needed, correction of coagulopathy often seen with HELLP syndrome, and serial imaging and LFT assays. ${ }^{2}$ Platelets and blood are repleted as needed based on the complete blood count. Many advocate for the use of corticosteroids as well. Historically, it was thought that the mainstay of treatment for HELLP syndrome should be immediate delivery. There is now evidence that stabilizing these patients first with antepartum corticosteroids can improve outcomes and reduce maternal and fetal morbidities and mortalities. ${ }^{5}$ Not all studies agree on this point, however. It is clear that corticosteroids can improve laboratory values such as platelet counts and liver enzymes, but this does not always equate improved maternal outcomes. A systematic review of the literature from 2015 sets out to investigate the overall impact of corticosteroids on women with HELLP syndrome, and ultimately found that it improves platelet count and serum lactate dehydrogenase. These clinical outcomes reduced hospital stay and ICU admissions, but did not necessarily correlate to maternal survival or decreased morbidity. ${ }^{6}$ Corticosteroids are still used by many physicians in the treatment of HELLP syndrome, especially when plasmapheresis is being performed.

Many recognize the utility of plasma exchange in the treatment of HELLP syndrome, noting its significant reduction in maternal mortality. A study from Turkey in 2005 treated 29 patients with HELLP syndrome and compared them with 26 patients in the control group. Maternal mortality was $23.1 \%$ in the control group, with no deaths in the treatment arm. The patients treated with plasma exchange also experienced more rapid improvement of their laboratory abnormalities including platelet count, AST, ALT, and lactate. ${ }^{7}$ Plasmapheresis is sometimes reserved only for patients with HELLP syndrome refractory to other supportive treatments, or those who have developed DIC. In women who have been treated with platelet transfusions without improvement of their disease, plasmapheresis is often indicated. One case report discusses the use of plasma exchange therapy in the treatment of hepatic infarction in the setting of HELLP syndrome, as well as serial imaging studies. $^{8}$ Corticosteroids are often used during plasma exchange to prevent the development of new autoantibodies, which are actively being filtered out by the plasma exchange procedure.

Aside from laboratory abnormalities and DIC, another catastrophic complication of HELLP syndrome is subcapsular liver hematoma. Hematomas of this nature can rupture, which is a rare but fatal occurrence. In addition to avoiding direct manipulation with the liver, either during physical examinations or during cesarean section, special care must be taken with abdominal imaging when there is concern for hepatic injury. Although studies have shown that imaging abnormalities are rarely present in HELLP syndrome, when they are present, they can help dictate clinical decision making. ${ }^{9}$ Preferred imaging studies include ultrasound, CT, magnetic resonance imaging, or angiography. Abdominal CT is believed to be more sensitive than ultrasound for recognizing hematomas and the true extent of any hepatic hemorrhage. ${ }^{10}$ Serial imaging can help detect a growing hepatic hematoma, and can track any worsening necrosis of the liver. Additionally, imaging can detect abnormalities in the liver that may not be evident in laboratory testing. It is recommended to obtain imaging of the liver if HELLP syndrome is suspected, regardless of the liver enzyme tests. Specifically, patients with right upper quadrant pain, neck pain, shoulder pain, or relapsing hypotension should undergo imaging. ${ }^{11}$ In the case of our patient, serial CT imaging was used to monitor the infarcted left lobe of the liver while the patient was being worked up by the transplant team for potential surgery.

Our case presents a young patient with no prior medical comorbidities in a low-risk pregnancy who developed a hepatic infarction in the setting of HELLP syndrome. The patient fortunately recovered her liver function and is expected to regenerate much of her hepatic tissue; however, we cannot yet know if she endured lasting hepatic injury or diminished function throughout her life. Further research into this area is needed to determine the long-term outcomes of women with liver injury related to pregnancy complications to better assist in patient counseling and education.

\section{Conflict of Interest}

None. 


\section{References}

1 Tufano A, Coppola A, Maruotti GM, Martinelli P, Cerbone AM, Di Minno G. HELLP syndrome and its relation with the antiphospholipid syndrome. Blood Transfus 2014;12(01):114-118

2 Suzumori N, Obayashi S, Kumagai K, Goto S, Yoshida A, SugiuraOgasawara M. A case of microangiopathic antiphospholipidassociated syndromes during pregnancy: review of the literature. Case Rep Med 2012;2012:827543

3 Lam MTC, Dierking E. Intensive care unit issues in eclampsia and HELLP syndrome. Int J Crit Illn Inj Sci 2017;7(03):136-141

4 Mikolajczyk AE, Renz J, Diaz G, Alpert L, Hart J, Te HS. Massive hepatic infarction caused by HELLP syndrome. ACG Case Rep J 2017;4:e81

5 Heller CS, Elliott JP. High-order multiple pregnancies complicated by HELLP syndrome. A report of four cases with corticosteroid therapy to prolong gestation. J Reprod Med 1997;42(11): 743-746

6 Mao M, Chen C. Corticosteroid therapy for management of hemolysis, elevated liver enzymes, and low platelet count (HELLP) syndrome: a meta-analysis. Med Sci Monit 2015;21:3777-3783
7 Eser B, Guven M, Unal A, et al. The role of plasma exchange in HELLP syndrome. Clin Appl Thromb Hemost 2005;11(02): 211-217

8 Hammoud GM, Ibdah JA. Preeclampsia-induced liver dysfunction, HELLP syndrome, and acute fatty liver of pregnancy. Clin Liver Dis 2014;4(03):69-73

9 Levine H, Zarghouni M, Cannon W. Imaging manifestations of a dreaded obstetric complication in the immediate postpartum period. Proc Bayl Univ Med Cent 2014;27(02):141-142

10 Chou M-M, Chen Y-F, Kung H-F, et al. Extensive hepatic infarction in severe preeclampsia as part of the HELLP syndrome (hemolysis, elevated liver enzymes, and low platelets): evolution of CT findings and successful treatment with plasma exchange therapy. Taiwan J Obstet Gynecol 2012;51(03):418-420

11 Barton JR, Sibai BM. Hepatic imaging in HELLP syndrome (hemolysis, elevated liver enzymes, and low platelet count). Am J Obstet Gynecol 1996;174(06):1820-1825, discussion 1825-1827

12 Han X, Fan Y, Yu Y. Two cases of severe preeclampsia were diagnosed with HELLP postpartum after caesarian section. Case Rep Obstet Gynecol 2014;2014:747510 